\title{
La filosofía y la sociedad en el México actual
}

Gabriel Vargas Lozano

Departamento de Filosofía de la UAM-I

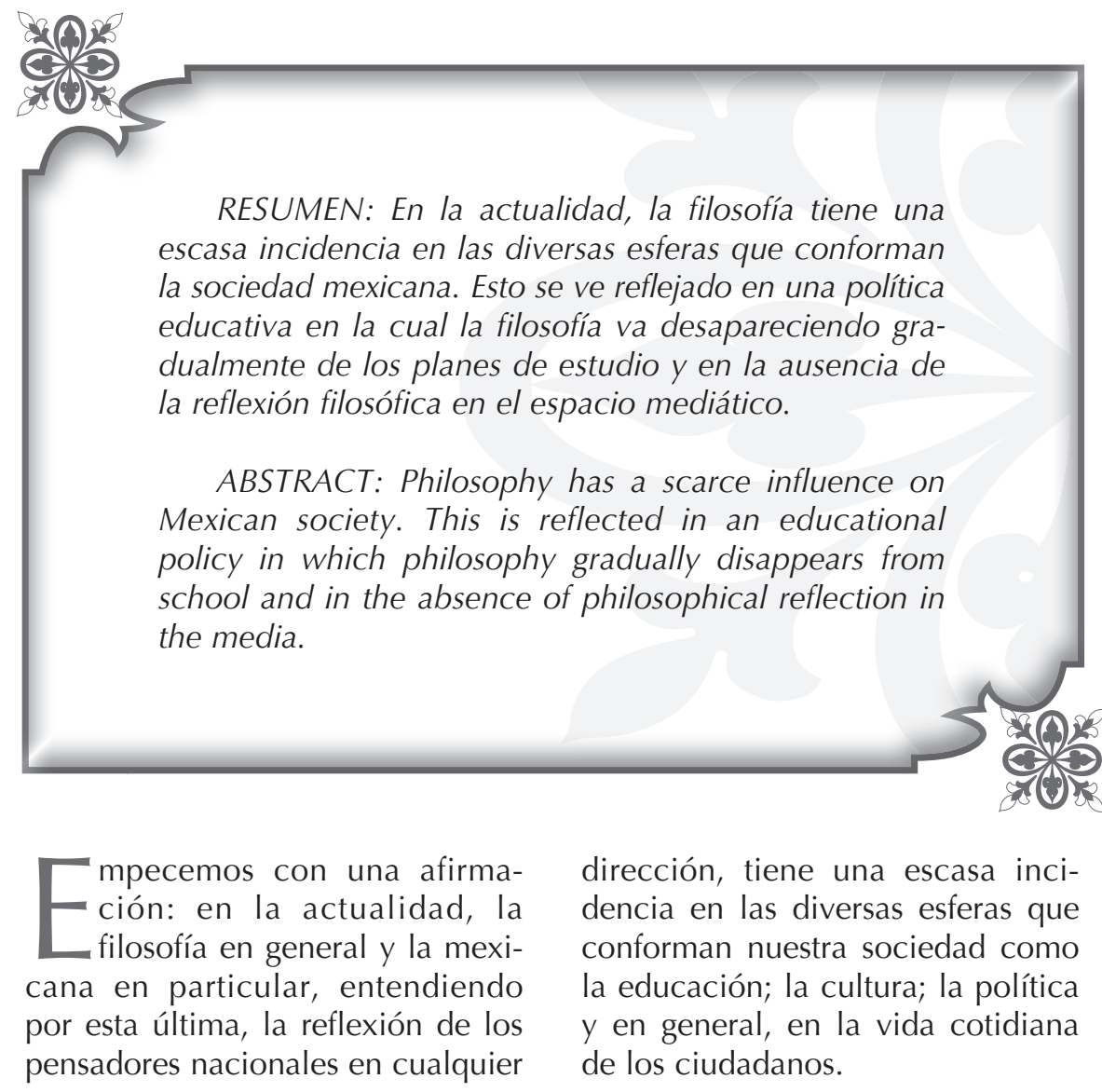


Este hecho debería causar extrañeza debido a que en la actualidad, la humanidad en su conjunto, está enfrentando una serie de graves problemas económicos, sociales, políticos, ambientales, ideológicos y científicos que requieren la intervención de todas las disciplinas pero en especial, de la filosofía y aún más, nos encontramos en un período histórico de transición hacia una nueva etapa llamada, a falta de otro nombre, posmodernidad o transmodernidad cuyas características (negativas y positivas) ya empiezan a aparecer en la situación actual. Si la filosofía ha tenido la función de orientar la vida hacia un mundo mejor, debería estar presente en forma muy visible; debería estar formando parte del debate público y sin embargo, ante los ojos del ciudadano común, aparece como una disciplina extraña cuando no, inútil y por tanto, innecesaria.

\section{El Estado mexicano frente a la filosofía}

Lo curioso es que la anterior no es sólo una opinión de un ciudadano que han mantenido en la des-ilustración sino también de algunos funcionarios del Gobierno. Recuerdo que el subsecretario del trabajo del sexenio de Vicente Fox (2000-2006), en una declaración periodística, expresó que el sistema no requería filósofos sino técnicos y que los primeros eran como los luditas, es decir, aquel movimiento que se opuso al progreso destruyendo las máquinas. El subsecretario tuvo una enérgica respuesta pública de mi parte que fue secundada públicamente por una serie de colegas. Su respuesta fue el silencio. ${ }^{1}$

\subsection{Política educativa}

Esta concepción negativa de la función de la filosofía por parte de los funcionarios del gobierno ${ }^{2}$ se ha traducido en una política educativa del Estado. En efecto, mientras el gobierno, durante cincuenta años (desde los años veinte hasta los setenta del siglo XX) fomentó el desarrollo de la filosofía otorgándole un lugar importante en la formación de los estudiantes de bachillerato y propiciando la fundación de Escuelas, Facultades e Institutos en diversas zonas del país (preferentemente del centro y del norte) ${ }^{3}$ y se desarrolló, por parte de una corriente de filósofos (en particular la latinoamericanista) una crítica a la imitación y a la dependencia'; a partir de 1985, se inició una tendencia que pretende reducir a la filosofía a su mínima expresión. En este sentido se operaron las modificaciones de los planes de estudio de los Colegios de bachilleres; las Preparatorias (a excepción de la UNAM) y el Colegio de Ciencias y Humanidades de la UNAM. Esta tendencia se expresó también en el intento, afortunadamente fracasado, por la reacción en contra de estudiantes y profesores, 
de desaparición de las licenciaturas de filosofía en la Facultad de Filosofía y Letras de la UNAM y la sustitución por un bachelor of arts mediante una reforma propuesta por el Rector Francisco Barnés de Castro. Finalmente, podemos decir que, salvo algunas notables excepciones, las Escuelas y Facultades de los Estados tienen un apoyo presupuestal mínimo para la realización de actividades de formación de profesores o investigadores ${ }^{5}$.

\subsection{La filosofía en los medios masivos de comunicación}

Finalmente, la filosofía no tiene ningún lugar en los medios masivos de comunicación impresos o electrónicos. Ello no quiere decir que, de vez en cuando, se publiquen reseñas de libros o comentarios en los suplementos culturales o que se mantenga, con un gran esfuerzo, algún programa radiofónico ${ }^{6}$ pero la verdad es que los dos canales culturales (Canal 11 y Canal 22) no otorgan ningún espacio a la filosofía producida por los nacionales.

\subsection{Causas del fenómeno}

A mi juicio, las causas de fondo provienen, por un lado, de una tendencia general del sistema capitalista; que se ha intensificado en las últimas décadas y que afecta a todos los países, sin embargo, esta tendencia alcanza su límite crítico en sociedades como la mexicana cuyos gobiernos han profundizado la dependencia en todos los ámbitos como una "solución" a la crisis por la que atravesamos.

\subsubsection{Lugar de la filosofía en la estructura social}

Con relación al primer aspecto, podemos decir que el sistema capitalista se basa, entre otras, en dos tendencias principales; por un lado, la mercantilización de todas las actividades humanas (las ideas, los cuerpos, la política, el arte, la religión, la ciencia y muchas otras) y por otro, la intensificación de los procesos de alienación o enajenación pública. En efecto, si la filosofía tiene una función altamente humanista, crítica y desalienadora, entonces sus disciplinas, de acuerdo a la "racionalidad del sistema", no deberían formar parte de la actividad de los ciudadanos, a menos que pudieran favorecer esta tendencia.

\subsubsection{La ideología y la enajenación}

La mercantilización está vinculada profundamente con las ideologías y el proceso de enajenación que tienen el objetivo de tratar de encubrir en la conciencia de los individuos, las condiciones de cosificación e injusticia intentando que los individuos las consideren "naturales" e "inevitables". Para cumplir esta función, el sistema de dominación ha encontrado, en los medios masivos de comunicación, un excelente instrumento para la 
manipulación de las conciencias. Para lograr este propósito, ha utilizado disciplinas como la psicología, la sociología y la neurobiología, para impedir un pensamiento libre y crítico y orientarlo hacia la creación de mundos ficticios. Esta tendencia se inició desde los años 20 (¡y todavía no se había inventado la televisión!) por Edward Louis Bernays, hijo de Anna Freud, hermana de Sigmund Freud, quien estudió los mecanismos psicológicos que deberían ser empleados en el mercado y la política. ${ }^{7}$

Por tanto, es fácil comprender la causa por la cuál los filósofos han sido desplazados del espacio público y reducidos a la Universidad y dentro de la Universidad a los Departamentos, Facultades o Institutos de filosofía. ${ }^{8}$

Pero además, el uso intensivo de los medios audiovisuales en la información y comunicación desplazaron la letra escrita por la imagen y esta conversión fue aprovechada por el sistema para sustituir la argumentación racional por la percepción sensorial. Ello no quiere decir que las imágenes, bien utilizadas, no puedan servir también de un extraordinario instrumento pedagógico para la ciencia o la filosofía sino que el sistema aprovechó dicha conversión para la creación de un mundo imaginario en donde solo tiene cabida lo que Ilamaba Karel Kosik, "el mundo de la pseudoconcreción".9 Por tanto, los filósofos, ya no son los que proponen una orientación al conjunto social (con excepción de los que el sistema selecciona y aprueba) sino el conductor de radio o televisión que orienta toda la información de acuerdo a los intereses de los propietarios de los medios masivos de comunicación. La razón de ello se encuadra en su propia lógica: desde el punto de vista del sistema, la filosofía ha sido, por lo general, crítica de los poderes establecidos; induce a la reflexión y convoca a la argumentación lógica mientras el sistema de dominio busca impactar los sentidos para la enajenación y la manipulación del televidente o radio-escucha. Es por ello que está interesado en un espectador acrítico, un hombre unidimensional (Marcuse) al que luego le adjudica una "forma de ser"; un "rechazo natural" del ciudadano hacia todo lo que signifique pensar.

Se trata entonces de dos lógicas encontradas. Si la filosofía es, por excelencia, la disciplina que permite que los individuos se pregunten por el significado y sentido de la vida y el medio privilegiado para pensar la racionalidad y la justicia de una sociedad o del mundo; si la filosofía permite que los ciudadanos se vuelvan más conscientes de sí mismos, entonces la filosofía no se encuentra dentro de la lógica de la mercantilización y del dominio. 
Pero si lo anterior es correcto ¿cómo es que siguen existiendo las escuelas de filosofía; los profesores e investigadores y las publicaciones? a causa es que, por fortuna, existen dentro del sistema una serie de contradicciones que permiten la subsistencia de la filosofía. El sistema establece una determinada dinámica para propiciar su desarrollo, sin embargo, el mundo de la vida es más complejo, más rico y afortunadamente más rebelde frente a las normas impuestas.

Pero además, agreguemos que, a través de su larga historia, la filosofía ha logrado obtener un lugar como parte constituyente de los sistemas o como crítica de él. Un claro ejemplo de esto fue la polémica entre Ginés de Sepúlveda y Bartolomé de las Casas sobre la humanidad de los indígenas. Mientras Ginés, armado del aristotélico-tomismo legitimaba la dominación española en América, Bartolomé, como una voz solitaria, defendía a los indígenas, a pesar de que clamara en el desierto. Así que dentro de los sistemas más absolutistas se presentan contradicciones y coyunturas que permiten la subsistencia de la filosofía, aunque también es cierto que hay períodos en los que toda reflexión filosófica crítica es eliminada junto a sus autores. Esto ocurrió en el nazismo; en el estalinismo o en las dictaduras sangrientas de América del Sur durante las décadas de los setenta y ochenta del siglo pasado y que expulsaron de sus países a los filósofos críticos. $^{10}$ Agreguemos que existe una diferencia específica entre el desarrollo de la filosofía en Europa y en América Latina. Mientras en el viejo continente ha existido una fuerte tradición filosófica, en nuestros países, se ha presentado de manera fracturada y su desarroIlo plural sólo se ha experimentado hasta el siglo XX.

Pero, además, el arraigo de la filosofía en los países europeos proviene también de la participación de los filósofos en la construcción de sus sociedades. El ejemplo clásico es la extraordinaria aportación de los filósofos ilustrados que destruyeron las bases teóricas del absolutismo y prefiguraron las nuevas instituciones de la modernidad. En cambio, en nuestros países, nuestros filósofos, con notables excepciones, no se han encontrado a la altura de los acontecimientos históricos. Un ejemplo de ello fue la falta de una reflexión filosófica específica durante la crisis que condujo a la Revolución Mexicana de 1910. La justificación a posteriori que hicieron Antonio Caso y José Vasconcelos en torno a la tesis de que su lucha en contra del positivo y a favor de un espiritualismo había sido antecedente de dicha Revolución, a mi juicio no se sostiene ya que se limitó a la crítica del positivismo (con el apoyo del Estado porfiriano) y no, como ocurrió en la Revolución Francesa, a la crítica del régimen porfiriano o a la caracterización de las instituciones del futuro. 


\section{을 Por tal motivo, una tradición tan fuerte como la Europea no es fácil de eliminar, a pesar de la tendencia del sistema hacia la li- quidación de toda forma de pensar crítica y de periodos históricos de totalitarismo.}

\subsubsection{La introducción del neolibe- ralismo}

Pero existe otro aspecto fundamental que ha afectado al desarrollo de toda la vida social en México y a la filosofía en particular. Trataré de explicarlo en forma breve: en la década de los ochenta, los gobiernos de Ronald Reagan (EUA) y Margaret Thatcher (Gran Bretaña) junto a agencias como el Banco Mundial y el Fondo Monetario Internacional, establecieron la política neoliberal basada en las teorías de Milton Friedman y Friedrich von Hayek. Esta estrategia se basó en el desmantelamiento del Estado benefactor (welfare state); la disminución del papel del Estado y la privatización de las empresas públicas, entre otras medidas. En nuestro país, esta estrategia fue adoptada por el régimen de Miguel de la Madrid (1982-88); profundizada por Carlos Salinas de Gortari (1988-1994) y continuada por los demás gobiernos hasta la fecha. ¿Por qué se adoptó dicha estrategia? Una de las causas fue la desastrosa política petrolera de José López Portillo (1976-82) y que desembocó en la crisis al final del sexenio pero otra fue la decisión del grupo gobernante de convertir la economía nacional en un sector subordinado de la economía norteamericana. Este hecho implicó un cambio de la política en todos los órdenes. Así, mientras en los dos primeros tercios del siglo XX se pretendió construir un país con un desarrollo propio en lo económico, lo político y lo cultural, a partir de aquel momento, se consideró que el país tenía que adoptar una posición dependiente con respecto a las necesidades e intereses de los países altamente industrializados. Ya no se trataba de sustituir importaciones sino ubicarse en forma subsidiaria en el sistema global. Por tanto, a los ciudadanos había que formarlos en el uso de las nuevas tecnologías; adiestrarlos para insertarse en los servicios y para la reproducción acrítica de las teorías. Esta concepción de fondo es, desde mi punto de vista, la causa de las opiniones de los funcionarios gubernamentales sobre la filosofía.

Agregaría que los filósofos podrían haber mostrado que aún, en la lógica del sistema, la filosofía podía cumplir un papel importante en la conformación de un individuo con una mentalidad más organizada e ilustrada a través de la educación filosófica. Tirios y troyanos, podrían haber encontrado, en la página de web de la Asociación Filosófica Americana (APA) del país más pragmático del mundo, los Estados Unidos, los elementos para demostrar que la filosofía puede ser útil para el desempeño de todas las 
actividades pero nuestra comunidad filosófica permaneció en silencio.

Hasta aquí, el lugar que, en mi opinión, asigna a la filosofía el actual sistema en nuestro país.
Ahora veamos como ha reaccionado nuestra filosofía frente al devenir del sistema en estos años.

\section{La filosofía mexicana frente a la sociedad}

A mi juicio, prácticamente todos los filósofos mexicanos han tenido una idea de lo que ha sido, es y podría ser el país. Lo que ocurre es que no siempre la han hecho expresa y tampoco han estado de acuerdo sobre la forma en que la filosofía puede tomar a su cargo la problemática nacional. Por ejemplo, en el debate de los sesenta entre Leopoldo Zea y quienes propiciaron la filosofía analítica (Luis Villoro, Fernando Salmerón y Alejandro Rossi, principalmente) se encontraban no sólo diversas formas de entender a la filosofía y sus relaciones con la sociedad sino una forma de entender el destino de México. Zea criticó el modo inauténtico de hacer filosofía a partir de la reproducción acrítica de los sistemas y abogó porque la filosofía enfrentara directamente nuestros propios problemas desde una concepción historicista, sin embargo, no hizo una crítica sistemática al corporativismo y en general al sistema autoritario de dominación. Luis Villoro, en cambio, consideró, en los setenta, que el país lograría convertirse en una potencia media y que requería una filosofía fundada en la ciencia y la técnica que tuviera una fun- ción metateórica. ${ }^{11}$ Años después pudimos comprobar que el país no avanzaría por esa senda y que, por el contrario, nos encontrábamos en una severa crisis económica y social. Ello no quiere decir que no fueran válidas, en sí mismas, varias de las posturas de nuestros dos importantes filósofos. Yo creo que hoy nadie podría rechazar la necesidad de que la filosofía enfrente los grandes problemas que nos aquejan y tampoco la necesidad de una filosofía del lenguaje o una filosofía de la ciencia y de la técnica pero detrás de todo ello existía (y existe) el tema de la relación entre filosofía y sociedad. ${ }^{12}$

Pero la pregunta es: ¿cuál fue la reacción de los filósofos mexicanos ante la estrategia neoliberal?

En lo que se refiere a las tendencias de la investigación, la reacción ha sido diversa y compleja. Por un lado, los filósofos han tenido que someterse a una lógica productivista impuesta a las Universidades por los últimos regímenes, sin embargo, por otra, han gozado de libertad de investigación debido a dos causas: la tradición de la autonomía univer- 
sitaria que ha sido preservada en las Universidades públicas y la escasa incidencia de sus reflexiones en la vida pública.

Aquí entramos al terreno de la filosofía misma en donde se combinan impulsos provenientes, por así decirlo, de su exterior e impulsos internos.

Un recuento esquemático nos arrojaría el siguiente resultado:

En primer lugar, continúa la tradición de importación de corrientes filosóficas "a la moda" en Europa o los Estados Unidos. Este hecho es inevitable y no tendría mayor significado si: a) la importación se practicara en forma creativa y b) se utilizara a las grandes corrientes del pensamiento para aportar un conocimiento para nuestras sociedades.

En segundo lugar, se han abordado temáticas importantes como las de la modernidad y la posmodernidad; feminismo, el multiculturalismo; teorías de la acción comunicativa y hermenéutica. Esta última ha sido la que más ha Ilamado la atención en los últimos tiempos, en sus diferentes versiones, a partir de la crisis del intento de lograr una filosofía cientificista y plenamente objetiva.

En tercer lugar, se ha registrado un giro pragmático hacia el análisis de temas específicos que también importarían a la sociedad en su conjunto como: el aborto ${ }^{13}$, la equidad de género, la eutanasia, la pena de muerte, los problemas ecológicos; la relación entre ética y política y la bio-ética.

En cuarto lugar, a partir del pacto político entre el gobierno y las fuerzas de oposición, en 1977, ha resurgido, de manera significativa, la problemática de la filosofía política abordando la democracia, los derechos humanos, las ideologías, las formas de Estado, etc.

Pero, en quinto lugar, considero que también es de interés el hecho de que la "filosofía mexicana"14 se ha dedicado, de manera creciente, al rescate de nuestro pensamiento y de nuestras tradiciones culturales, renovando los estudios de historia de la filosofía en México y en Latinoamérica y ubicando nuestra reflexión filosófica en relación con el entorno cultural. Por mi lado, considero que debemos hacer filosofía, en sentido estricto del término, pero enclavada profundamente en nuestra realidad. Esta es una característica clásica de la filosofía: Aristóteles reflexionó sobre temas ontológicos y gnoseológicos pero también sobre Atenas desde el punto de vista ético y político y buscó soluciones concretas para su sociedad; Locke reflexionó sobre el tema del conocimiento pero también innovó en la filosofía política proponiendo el iusnaturalismo, justamente cuando el rey había dejado el poder (recuérdense sus Dos ensayos sobre 
el gobierno civil); Hegel pensó la relación entre el ser y la nada, en su Ciencia de la lógica pero en su Filosofía del derecho, no sólo se deslindó de la tradición democrática de Rousseau y del absolutismo proponiendo un Estado racional sino que avanzó en los conceptos de sociedad civil y eticidad. Insisto: la filosofía clásica se mueve entre lo universal y lo particular, entonces, la pregunta que surge es: ipor qué, en nuestro país y en otras naciones latinoamericanas, se escinde en forma abismal lo universal de lo particular? La respuesta a esta pregunta podría ser abordada desde tres dimensiones: a) se busca desarrollar un tipo de filosofía concentrada en aspectos universales que, por lo general, implican la aclaración o profundización de un filósofo sin tomar en cuenta sus implicaciones sociales; b) se reflexiona sobre alguna temática pero sin extraer sus consecuencias para la humanidad; c) se elude el compromiso social y ético del filósofo. En torno a este último aspecto, uno de los casos más graves es, por ejemplo, el de Martín Heidegger. Por un lado, nadie puede poner en duda la importancia de sus reflexiones filosóficas pero ¿qué opinión nos merece el hecho de que no hubiera dicho absolutamente nada sobre el nazismo y en especial sobre el genocidio de millones de judíos y personas de otras nacionalidades en los hornos crematorios? $Y$ de igual manera, para poner un ejemplo más cercano, ¿el filósofo mexicano puede quedarse callado ante las condiciones de pobreza, desigualdad, injusticia y corrupción en que nos encontramos? ¿No debería pensar, como los clásicos, en la relación existente entre la filosofía que practica y la sociedad en que vive?

Quiero aclarar que, a mi juicio, el filósofo tiene el derecho de reflexionar sobre lo que desee y desde la perspectiva que elija, con la condición de que sea creativo y no meramente repetitivo pero, a mi juicio, no puede obviar que su reflexión está situada en una sociedad que ha pasado por trescientos años de colonia y que, en muchos sentidos, sigue viviendo en condiciones de dependencia. A mi juicio, el filósofo mexicano o latinoamericano debería tomar explícitamente una posición al respecto..

En suma, mi conclusión es que, por un lado, el sistema, sobre todo en los últimos años, ha hecho esfuerzos por limitar a la filosofía. A pesar de ello, una parte de nuestra comunidad ha tratado de responder, a su manera, a las necesidades generales de la sociedad, sin embargo, sus esfuerzos han sido insuficientes. 


\section{3. ¿Qué podemos hacer?}

La pregunta entonces es iqué podemos hacer para que la reflexión filosófica cumpla una función en la sociedad mexicana?

En primer lugar, considero que no deberíamos entender a la filosofía como una disciplina que debería ser cultivada exclusivamente en las Escuelas, Facultades o Institutos de filosofía sino como una disciplina que debe ser cultivada en todos los ámbitos de la sociedad. Ello implica, por supuesto, diversos problemas específicos que deben ser afrontados. Uno de ellos es el de la forma en que la filosofía puede relacionarse con la sociedad ${ }^{15}$ y otro es el de la función de la filosofía en diversos ámbitos. En efecto, en el libro Philosophy: school of freedom, que es un importante diagnóstico mundial del estado de la filosofía, esta disciplina debe ser enseñada a los niños; a los adolescentes; a los adultos y a los mayores y desde luego, en la secundaria; la preparatoria; la universidad y a los ciudadanos en la plaza pública.

En este sentido, podemos afirmar que en nuestro país no ha habido un plan de desarrollo de la filosofía en todos los niveles como lo recomienda la UNESCO. Para poder llevar a cabo esta labor se requiere exigir a las autoridades educativas del Estado (SEP, CONACYT, CONACULTA, editoriales como el FCE, Comisiones de cultura de las
Cámaras de diputados y senadores; Universidades y otras instituciones culturales) que den el espacio que se merece a la filosofía.

En segundo lugar, se requiere una nueva actitud del filósofo. En esta dirección, en una importante reunión celebrada en Dakar en enero de 2006 por parte de la UNESCO y la FISP (siglas en francés de la Federación Internacional de Sociedades de Filosofía) denominada "Philosophy teaching in the context of globalization" se pedía a los filósofos "dejar su "espléndida soledad" que a veces se separa de las realidades del mundo, para confrontar los problemas reales experimentados por hombre y mujeres y contribuye a encontrar respuestas para ellos"16

En tercer lugar, se requiere poner en marcha una reforma de la enseñanza de la filosofía en las escuelas de filosofía en el país. Para ello, me permito hacer cuatro sugerencias: ${ }^{17}$

1) La falta de cursos de didáctica de la filosofía. Si el estudiante egresará como profesor, en su carrera no recibe ninguna formación en este sentido. Aún más, se ha descuidado, salvo en la materia de lógica, el estudio de la didáctica de nuestra disciplina y que hoy debería incluir el uso de las nuevas tecnologías. 
2) Los cursos de filosofía en México vinieron a menos porque no se renovó su contenido y metodología. Hoy observamos en nuestro país un creciente interés por parte de diversos investigadores para realizar la necesaria historia de la filosofía en México pero no se ha traducido en su fortalecimiento en los planes de estudio ni en la enseñanza. Aún hoy existen muchos profesores que no incorporan en sus materias el estudio de nuestros filósofos y que no han tomado conciencia de la necesidad de la disciplina para desarrollar una reflexión que tenga conciencia de que la filosofía tiene que responder a nuestros propios problemas independientemente de su posible universalización. La historia de la filosofía en México implica la recuperación de nuestras tradiciones; el hacer valer las aportaciones de nuestros pensadores y en vincular el estudio de la filosofía en general con los problemas de nuestro país; nuestro continente e inclusive con la filosofía en lengua española.

3) En los planes de estudio de las carreras de filosofía, no se forman los especialistas necesarios para establecer las relaciones pertinentes entre la filosofía y las diversas carreras que se enseñan en la Universidad. Por ende, tampoco existen en esas carreras, los espacios necesarios para establecer un vínculo productivo. ${ }^{18}$ Creo que los especialistas saben que la filosofía ha tenido históricamente y tiene actualmente una relación con la economía; la ciencia política; la historia; la medicina; la física; la biología; la ingeniería; la arquitectura, etc. Lamentablemente, los pocos espacios existentes se desaprovechan y he conocido que en la carrera de economía, algún colega, en lugar de enseñar la relación entre Adam Smith, Karl Marx hasta Hayek pasando por Joan Robinson entre filosofía y economía, les enseñaba El ser y el tiempo de Martín Heidegger.

Este desencuentro entre filosofía y ciencia implicó también el desencuentro entre ciencia y filosofía.

Recordemos que en la ciencia misma se produjo un rechazo de la filosofía por su carácter especulativo. Dos ejemplos los podemos encontrar en la Historia y en la ciencia política: en la historia se impuso la tendencia empirista en contra de la especulativa de la filosofía clásica pero no se avanzó en la reflexión sobre los problemas del conocimiento histórico por parte de los historiadores y muy poco por parte de los filósofos. En el caso de la ciencia política se adoptó el conductismo, 
pero ya en la década de los sesenta, Isaiah Berlin mostró la insuficiencia de un análisis conductista porque no respondía al problema del ciudadano: ¿por qué debo obedecer? Y los valores subyacentes. Se requería entonces rejuvenecer una disciplina que había sido dada por muerta: "la filosofía política".

4) Finalmente, la falta de espacios para el diálogo entre los que sostienen diversas corrientes filosóficas sobre temas comunes. Para ello se requiere que exista la voluntad de un debate que siga las normas que propone Jürgen Habermas para un "diálogo libre de dominio" y que sí podrían aplicarse en la educación.

En suma, la ausencia de recursos pedagógicos idóneos; la falta de relación entre la filosofía y nuestra propia tradición; la carencia de especialistas que permitan la intermediación con otras disciplinas y la falta de espacios dialógicos explica, en parte, la falta de comprensión sobre la función de la filosofía por parte de otros profesionales y su ausencia en el conjunto de la sociedad.

\section{Conclusión}

La filosofía mexicana, en su enseñanza, investigación y difusión ha sufrido el impacto del sistema de dominación que busca reducir su importancia en la educación del ciudadano. La comunidad filosófica, por su parte, ha tratado de responder, de diversos modos, a la situación por la que ha atravesado la sociedad mexicana, latinoamericana y universal. Pero, como hemos dicho, hace falta una mayor incidencia de la filosofía en todas las esferas de la sociedad mexicana.

Se requiere desarrollar todo un programa de ilustración pública (como de alguna manera ha estado haciendo la comunidad científica) que implique varias acciones: establecer una sintonía entre las necesidades sociales y la reflexión filosófica; hacer comprensible la reflexión filosófica a pesar de que se sabe que existen ciertos aspectos que requieren especialización pero, si el filósofo es conciente de su responsabilidad social, está obligado moralmente a "colocar una escalera" para que la gente pueda acceder a la comprensión de la disciplina independientemente de que en ella existen temas que también están a flor de tierra. Además, la comprensión general de la función que ha tenido la filosofía en todos los tiempos, redundará en la fortaleza de la propia actividad filosófica al tener un respaldo público. Esta labor debe ser producto de quienes se dedican a esta actividad ya que los medios masivos de comunicación, solo darán espacio a la filosofía si: implica algún beneficio económico, político o ideológico para el sistema o si el público lo demanda. ${ }^{19}$ 
$Y$ finalmente, se requiere utilizar los nuevos recursos que ofrece el internet y que a pesar de que se encuentran ya saturadas de propaganda comercial, no ha podido impedir, hasta ahora, la comunicación de millones de personas en todo el mundo, en formas extraordinariamente variadas. Aquí hay un camino para la difusión de la filosofía a un público más amplio. ${ }^{20}$

Hoy nos encontramos con una sociedad sumida en una profunda crisis. La filosofía, las ciencias naturales y sociales y las humanidades en su conjunto deberían estar buscando alternativas para su solución, profundizando el conocimiento de nuestro país; fortaleciendo la incipiente democracia y pensando una orientación más justa para nuestra sociedad.

NOTAS

1 Dejo constancia de mi carta al Correo Ilustrado de La Jornada, el 9 de febrero de 2004, titulada "La filosofía, necesaria en todas las profesiones y para alcanzar libertades": "Señora directora: Ayer fueron publicadas unas declaraciones del señor Francisco Xavier Salazar Sáenz, subsecretario de la Secretaría del Trabajo y Previsión Social --en torno a la filosofía y las humanidades- que son verdaderamente sorprendentes por su ignorancia y que no merecerían una respuesta si no representaran una peligrosa tendencia que se observa en la actualidad.
El señor Salazar dice que las instituciones de educación superior y el gobierno federal, en lugar de apoyar a la filosofía y las humanidades, "deberían alentar y explorar otras alternativas" técnicas como la ingeniería y la biotecnología, entre otras. Esta posición denota, en primer lugar, incomprensión sobre la función que tiene en la sociedad la filosofía en particular y las humanidades en general y, en segundo, la idea de que nuestro país debe convertirse en una empresa maquiladora movida por un ejército de robots donde los que "piensan" serían una elite ubicada en las grandes metrópolis. Para ilustración del señor Salazar, la filosofía es la única disciplina que puede proporcionar, a cualquier persona, una conciencia racional de su situación en el mundo, capacidad para resolver los problemas y para analizar conceptos y argumentos, desarrollo de la capacidad de expresión y comunicación, así como cultura necesaria para desempeñar, de mejor manera, cualquier trabajo. La filosofía es necesaria en todas las profesiones (desde la ingeniería hasta la medicina) porque sus disciplinas (la ética, estética, lógica, filosofía de la ciencia y la tecnología, la filosofía política, etcétera) proporcionan elementos necesarios para poder reflexionar sobre una serie de problemas que son de extrema importancia, como el significado y consecuencias de la automatización, la globalización, los nuevos descubrimientos de la ciencia, la desigualdad o la injusticia, por sólo citar algunos." 


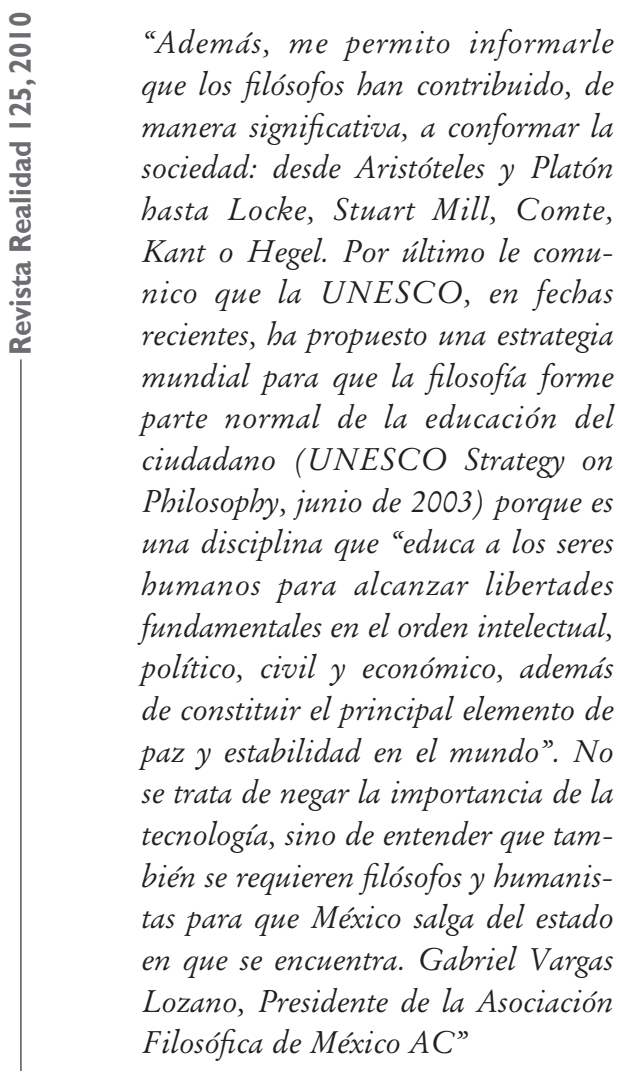

2 Incluiría aquí también a la Presidenta de CONACULTA del sexenio de Vicente Fox a quien no le interesaron las propuestas que hicimos llegar de parte del comité directivo de la Asociación Filosófica de México en 2002. Agregaría que la filosofía no figura como una de las ramas de la cultura que deberían ser apoyadas por ese organismo.

3 Las fechas son un indicador del interés del Estado en crear instituciones de enseñanza e investigación en filosofía: La Facultad de Filosofía y Letras de la Universidad Nacional de México, se funda el 23 de septiembre de 1924, sustituyendo a la Escuela Nacional de Altos Estudios. En 1940, se funda el Centro de estudios filosóficos de la Facultad de Filosofía y Letras de la UNAM (posteriormente convertido en Instituto de Investigaciones Filosóficas). En 1950 (21 de abril) se funda la Facultad de Filosofía, Ciencias y Letras, que se convierte en Facultad de Filosofía, Letras y Psicología en 1966 y desde 1974, Facultad de Filosofía y Letras, en la Universidad Autónoma de Nuevo León. En 1952 se funda la Facultad de filosofía de la Universidad de Guanajuato. El $1^{\circ}$ de febrero de 1956 se funda el Departamento de filosofía, hoy Facultad de Filosofía y Letras de la Universidad Veracruzana. El 15 de enero de 1957, se funda la Facultad de Filosofía y Letras de la Universidad de Guadalajara.1963. Se funda la Facultad de Filosofía y Letras de la Universidad Autónoma de Chihuahua.1965 (12 de mayo), se funda la Facultad de Filosofía y Letras de la Universidad Autónoma de Puebla. Ese mismo año se funda la Facultad de Humanidades de la Universidad Autónoma del Estado de México.

$4 \quad$ Ubiquemos aquí la línea que inició Antonio Caso contra lo que llamó la "imitación extralógica"; Samuel Ramos, en contra de la copia de corrientes y a favor de que la filosofía pensara a México (aunque no siempre con mucha fortuna); José Gaos, quien hizo un estudio a fondo de las características de la filosofía en los 
países productores y los dependientes; Salazar Bondy, quien hizo una crítica devastadora y pormenorizada (y no siempre justa) de la dependencia de la filosofía latinoamericana y Leopoldo Zea, quien criticó el eurocentrismo y el espíritu colonial.

5 Aquí el problema no ha sido sólo la falta de apoyos económicos sino también la ausencia de una estrategia conjunta para el desarrollo de la filosofía en todo el país.

6 Recientemente, un grupo de profesores de filosofía nos opusimos a la decisión, por parte del gobierno del Estado de Jalisco, de suprimir el único programa semanal dedicado a la filosofía por la Radio cultural del Estado (Mas información en mi blog: www.gabrielvargaslozano.org)

7 "The conscious and intelligent manipulation of the organized habits and opinions of the masses is an important element in democratic society. Those who manipulate this unseen mechanism of society constitute an invisible government which is the true ruling power of our country...We are governed, our minds are molded, our tastes formed, our ideas suggested, largely by men we have never heard of. This is a logical result of the way in which our democratic society is organized. Vast numbers of human beings must cooperate in this manner if they are to live together as a smoothly functioning society... In almost every act of our daily lives, whether in the sphere of politics or business, in our social conduct or our ethical thinking, we are dominated by the relatively small number of persons... who understand the mental processes and social patterns of the masses. It is they who pull the wires which control the public mind". Edward Louis Bernays en Propaganda. Estados Unidos, 1928

8 Este desplazamiento no sólo ha sido causado por el sistema sino por los mismos profesionales de la filosofía que, en algunos casos, declaran equivocadamente que la filosofía no tiene que ver con la sociedad o cuando la convierten en una reflexión exclusivamente técnica.

9 Karel Kosik, Dialéctica de lo concreto. Editorial Grijalbo, México, 1969.

10 Recordemos aquí a Marcuse, Horkheimer y Adorno que fueron expulsados de Alemania por el avance del nazismo; a Lukács o Kosik, quienes sufrieron persecución durante el estalinismo; a los filósofos españoles que fueron obligados a huir del franquismo o todos los filósofos latinoamericanos que se refugiaron en México durante las dictaduras de Guatemala, Argentina; Chile, Uruguay, Brasil y otros países.

11 Sobre los aspectos filosóficos de este debate, véase mi libro: Intervenciones filosóficas: ¿̇qué hacer con la filosofía en América Latina?. Ed. UAEM, México, 2007. 


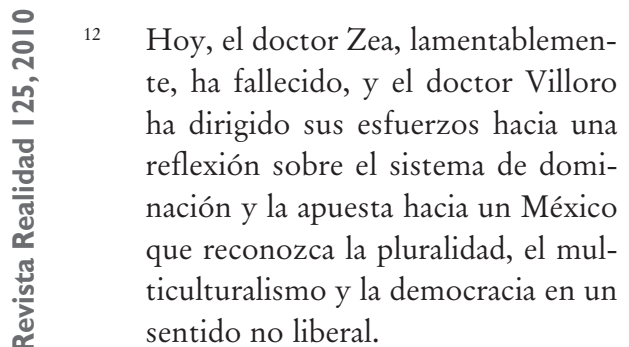

13 Véase la interesante propuesta de un grupo de investigadores del Instituto de Investigaciones Filosóficas de la UNAM que he reproducido en mi blog: www.gabrielvargaslozano.org

14 Sobre este punto, mi propuesta (que comparten otros colegas) es que debemos hablar de filosofía mexicana por el simple hecho de ser producida por nacionales y sin tener que comprometerse, necesariamente con la "filosofía del o de lo mexicano”. Además, habría que pensar si en Alemania se dice "filosofía en Alemania"; en Francia "filosofía en Francia” y en Inglaterra e Italia, “filosofía en esos países”.

15 Una, que extraigo del libro Philosophy. School of Fredom de la UNESCO: "por su verdadera naturaleza, la función esencial de la filosofía es extrapolar las estructuras teóricas que subyacen a los objetos culturales y extraer su vitalidad midiéndose a sí misma, contra los problemas concretos de las vidas de los pueblos y de las sociedades"; otra procedente de Filosofía de la praxis de Adolfo Sánchez Vázquez: la filosofía debe hacerse como una reflexión desde la praxis (no desde una teoría que contempla al mundo) que debe tener como centro la interpretación y la transformación del mundo.

Philosophy. A School of Freedom. Teaching philosophy and learning to philosophize: status and prospects. UNESCO, Paris, 207, p. 114

Para tratar de intercambiar experiencias, el licenciado Ricardo Villarreal y el que esto escribe, propiciamos la primera reunión de escuelas de filosofía del país en la Facultad de Filosofía y Letras de la Universidad Autónoma de Nuevo León, los días 28 y 29 de noviembre de 1991. Esta iniciativa continuó en 1992 en la Facultad de Humanidades de la UAEM; en 1993, en la Facultad de Filosofía y Letras de la Universidad de Guadalajara; en 1994, en el Departamento de filosofía de la Universidad Autónoma de Aguascalientes; en 1995, en la Facultad de Filosofía y Letras de la Benemérita Universidad Autónoma de Puebla; en 1996, en la Facultad de Filosofía de la Universidad Veracruzana y en 1997 en la Facultad de Filosofía y Letras de la UANL. Se conservan algunas memorias de las reuniones pero estas solo tuvieron un carácter deliberativo. En el prólogo a las memorias del Quinto encuentro, propuse que se hiciera un diagnóstico nacional de las fortalezas y debilidades de la enseñanza, la investigación y la difusión en filosofía que permitiera adoptar las medidas pertinentes. Hasta ahora no existe tal diagnóstico. 
La flexibilidad de los planes de estudio de las carreras, adoptado en la UAM-I, en fechas recientes, permite que los estudiantes cursen materias en otras disciplinas pero, por nuestro lado y creo que también por el suyo, se requiere formar a un especialista que sea capaz, por ejemplo, de establecer las relaciones correctas entre filosofía y biología; filosofía y física; filosofía e ingeniería; filosofía y medicina; filosofía y ciencias políticas, etc.
La UNESCO, a través del libro mencionado propone la creación de cafés filosóficos; talleres; consejeros filosóficos; realización de Olimpiadas; debates a partir de películas o de obras teatrales; blogs y páginas de internet; celebración de Día internacional de la filosofía y la impartición de la filosofía en múltiples ámbitos como la salud; la política y en los centros culturales.

En la UAM-I hemos creado la página de web: www.cefilibe.org y algunos maestros ya cuentan con páginas o blogs. 